\title{
Self-organized bosonic domain walls
}

\author{
Xingchuan Zhu, ${ }^{1}$ Shiying Dong, ${ }^{2}$ Yang Lin,,${ }^{2,3}$ Rubem Mondaini $\odot, 4,{ }^{4}$ Huaiming Guo, ${ }^{2, *}$ \\ Shiping Feng $\odot,{ }^{1}$ and Richard T. Scalettar ${ }^{5}$ \\ ${ }^{1}$ Department of Physics, Beijing Normal University, Beijing 100875, China \\ ${ }^{2}$ Department of Physics, Key Laboratory of Micro-Nano Measurement-Manipulation and Physics (Ministry of Education), \\ Beihang University, Beijing 100191, China \\ ${ }^{3}$ School of Applied Science, Beijing Information Science and Technology University, Beijing 100192, China \\ ${ }^{4}$ Beijing Computational Science Research Center, Beijing 100084, China \\ ${ }^{5}$ Physics Department, University of California, Davis, California 95616, USA
}

(Received 21 July 2019; revised manuscript received 18 December 2019; published 27 January 2020)

\begin{abstract}
Hard-core bosons on honeycomb lattice ribbons with zigzag edges are studied using exact numerical simulations. We map out the phase diagrams of ribbons with different widths, which contain superfluid and insulator phases at various fillings. We show that charge domain walls are energetically favorable, in sharp contrast to the more typical occupation of a set of sites on a single sublattice of the bipartite geometry at $\rho=\frac{1}{2}$ filling. This "self-organized domain wall" separates two charge-density-wave regions with opposite Berry curvatures. Associated with the change of topological properties, superfluid transport occurs down the domain wall. Our results provide a concrete context to observe bosonic topological phenomena and can be simulated experimentally using bosonic cold atoms trapped in designed optical lattices.
\end{abstract}

DOI: 10.1103/PhysRevResearch.2.013085

\section{INTRODUCTION}

One of the most interesting properties of condensed matter systems is their condensation into ordered low-temperature phases, breaking an underlying symmetry of the Hamiltonian. Such phases typically minimize the free energy $F$; coexistence of the distinct ordered patterns involves a domain wall, increasing $F$. Nevertheless, domain walls often exist in practice in experiments (or in simulations) as a consequence of long annealing times. This is especially the case in the presence of disorder which can pin their motion.

In addition to being manifest as metastable states, domain walls can also arise in other ways. An important example is provided by doping away from the commensurate antiferromagnetic (AF) filling of the cuprate superconductors [1], or the Hubbard and $t-J$ models that describe them [2-4]. Dopants do not spread uniformly, but instead form "charge stripes." Across these stripes there is a " $\pi$-phase shift" of the AF order [5]. The up-spin occupied sublattice interchanges across the stripe, realizing a domain wall.

In model Hamiltonian studies on "ladder" geometries using the density matrix renormalization group, the charge patterns are found to be "vertical stripes"; i.e., the doped holes lie parallel to the short direction of the cluster [3]. These charge patterns are fundamentally connected not only

\footnotetext{
*hmguo@buaa.edu.cn; rmondaini@csrc.ac.cn

Published by the American Physical Society under the terms of the Creative Commons Attribution 4.0 International license. Further distribution of this work must maintain attribution to the author(s) and the published article's title, journal citation, and DOI.
}

to magnetism, e.g., the $\pi$-phase shift, but also to chargedensity-wave (CDW) and $d$-wave pairing order. Studies of stripe physics and the associated domain walls remain of great interest [6,7], with the possible coexistence of Luther-Emery liquid states in which the spin excitations are gapped, and quasi-long-range superconducting correlations being a key issue [8].

We study bosonic particles on honeycomb ribbons. Four novel features emerge. First, we argue that charge domain walls are energetically favorable compared to occupation of a set of sites on a single sublattice of the bipartite geometry, even at half filling. This is a rather unique feature compared to situations in which domain walls are excitations rather than the ground state. Second, the low-density sites of the domain wall are arranged "horizontally" (parallel to the long axis), rather than vertically. These "self-organized domain walls" open the possibility of superfluid transport down the chain. Third, associated with this physics is a nontrivial Berry curvature, which changes sign across the domain wall. Finally, the system realizes an exotic one-dimensional supersolid.

\section{THE MODEL AND METHOD}

We consider spinless hard-core bosons on zigzag ribbons of a honeycomb lattice, described by the extended BoseHubbard model $[9,10]$ :

$$
H=-t \sum_{\langle i, j\rangle}\left(b_{i}^{\dagger} b_{j}+b_{j}^{\dagger} b_{i}\right)+V \sum_{\langle i, j\rangle} n_{i} n_{j}-\mu \sum_{i} n_{i} .
$$

Here $b_{i}\left(b_{i}^{\dagger}\right)$ are hard-core boson annihilation (creation) operators, and $n_{i}=b_{i}^{\dagger} b_{i}$ is the number operator. Hard-core bosons obey commutation relations $\left[b_{i}, b_{j}^{\dagger}\right]=0$ for sites $i \neq j$ and 


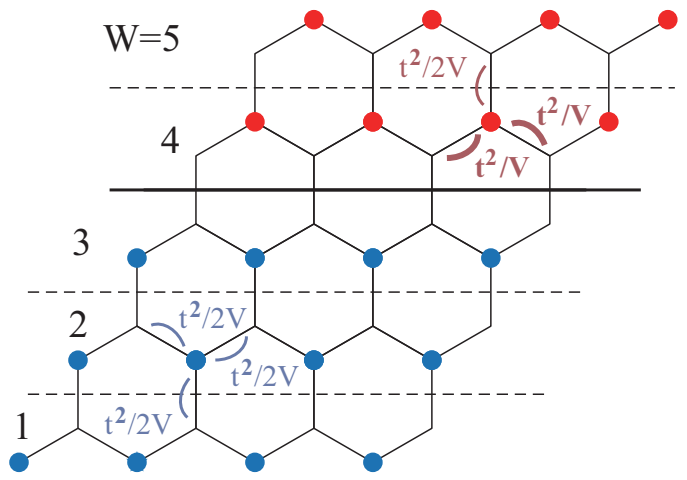

FIG. 1. Schematics of a width $W=5$ and length $L=4$ honeycomb lattice ribbon with zigzag edges. One of the (atomic limit) degenerate configurations of the $\rho=\frac{1}{2}$ insulator is shown. The filled circles represent hard-core bosons, with the colors distinguishing the sublattices. The domain wall is marked by the solid line. The dashed lines mark the domain walls of other degenerate configurations. The kinetic energy gains by second-order hopping processes are marked.

on-site anticommutation relations $\left\{b_{i}, b_{i}^{\dagger}\right\}=1$. The first term in Eq. (1) describes nearest-neighbor $(\mathrm{NN})$ hopping, with amplitude $t$ taken as the unit of energy $(t=1)$. The second term in Eq. (1) is the NN interaction $V$. Finally, $\mu$ denotes the chemical potential, which controls the number of bosons in the system. The model in Eq. (1) has a U(1) symmetry and is invariant under the transformation $b_{j} \rightarrow e^{i \theta} b_{j}$, where $\theta$ is a real-valued phase. This symmetry is spontaneously broken in a superfluid phase. The Hamiltonian is invariant under the inversion transformation center of honeycomb lattice ribbons with zigzag edges.

We employ the stochastic series expansion (SSE) quantum Monte Carlo (QMC) method [11] with directed loop updates to study Eq. (1). SSE expands the partition function in a power series and the trace is written as a sum of diagonal matrix elements. The directed loop updates and the fact that the discrete configuration space can be sampled without floatingpoint operations make the approach very efficient [12-14]. Our simulations are on finite lattices with the total number of sites $N=2 \times W \times L$, with $W$ the width and $L$ the length of a ribbon (see Fig. 1). The temperature is set to be low enough to obtain the ground-state properties. In calculating the eigenstates of interacting systems, we also use the exact diagonalization (ED) and density matrix renormalization group (DMRG) methods. The ED method is numerically exact, but has strong size limitation. By identifying a small subspace out of the exponentially large Hilbert space, the DMRG method can simulate larger systems, and the results are accurate up to a truncation error [15].

\section{BAND STRUCTURE}

The band structure of honeycomb lattice ribbons can be determined analytically. One fermion has exactly the same energy as one hard-core boson due to the absence of exchange statistics. For $W=2$, the Hamiltonian in momentum space is

$$
H_{2}\left(k_{x}\right)=\left[\begin{array}{cc}
h_{1}\left(k_{x}\right) & h_{2} \\
h_{2}^{\dagger} & h_{1}\left(k_{x}\right)
\end{array}\right],
$$

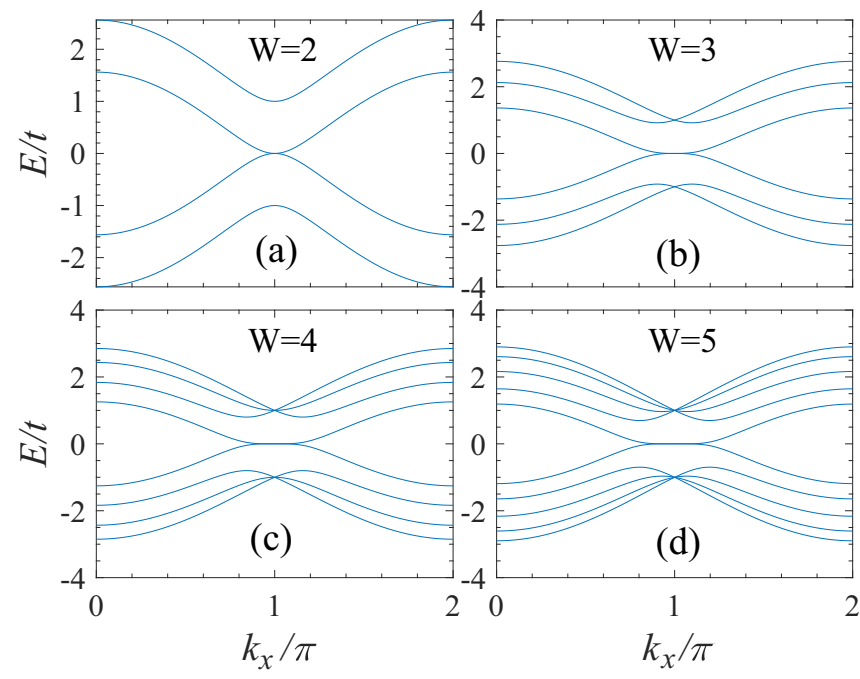

FIG. 2. The band structures of the honeycomb lattice ribbons with the widths (a) $W=2$, (b) $W=3$, (c) $W=4$, (d) $W=5$.

with $\gamma_{k}=1+e^{i k_{x}}$, and

$$
h_{1}\left(k_{x}\right)=\left(\begin{array}{cc}
0 & -t \gamma_{k} \\
-t \gamma_{k}^{*} & 0
\end{array}\right), \quad h_{2}=\left(\begin{array}{cc}
0 & 0 \\
-t & 0
\end{array}\right) .
$$

The energy spectrum contains four branches, $E_{i}=( \pm 1 \pm$ $\left.\sqrt{9+8 \cos k_{x}}\right) t / 2(i=1,2,3,4)$. The band bottom is located at $k_{x}=0$, and the corresponding eigenvalue is $-(1+$ $\sqrt{17}) t / 2$ (see Fig. 2). Thus the lower boundary of the phase diagram, where the density first begins to become nonzero, is a straight line $\mu / V=-\frac{1+\sqrt{17}}{2} t / V$.

Under a particle-hole transformation $b_{i}^{\dagger} \rightarrow h_{i}$, the Hamiltonian Eq. (1) becomes

$$
\begin{aligned}
H= & -t \sum_{\langle i, j\rangle}\left(h_{i}^{\dagger} h_{j}+\text { H.c. }\right)+V \sum_{\langle i, j\rangle} n_{i}^{h} n_{j}^{h} \\
& -(3 V-\mu) \sum_{i \in \text { bulk }} n_{i}^{h}-(2 V-\mu) \sum_{i \in \text { edge }} n_{i}^{h}+E_{0},
\end{aligned}
$$

where $n_{i}^{h}=h_{i}^{\dagger} h_{i}$ is the hole number operator and $E_{0}=$ $\frac{3}{2} V N-L V-\mu N$ is a constant. In momentum space, the hole Hamiltonian is

$$
H_{2}^{h}\left(k_{x}\right)=\left[\begin{array}{cccc}
-2 V & -t \gamma_{k} & 0 & 0 \\
-t \gamma_{k}^{*} & -3 V & -t & 0 \\
0 & -t & -3 V & -t \gamma_{k} \\
0 & 0 & -t \gamma_{k}^{*} & -2 V
\end{array}\right] .
$$

The energy spectrum $E_{1,2}=-\frac{t}{2}-\frac{5 V}{2} \pm \frac{1}{2} P_{k,+}, E_{3,4}=\frac{t}{2}-$ $\frac{5 V}{2} \pm \frac{1}{2} P_{k,-}$, with $P_{k, \pm}=\sqrt{9 t^{2}+8 t^{2} \cos k_{x}+V^{2} \pm 2 t V}$. The band bottom determines the upper boundary of the phase diagram, which is described by the curve $\frac{\mu}{V}=\frac{1}{2} \frac{t}{V}+$ $\frac{1}{2} \sqrt{17 \frac{t^{2}}{V^{2}}+2 \frac{t}{V}+1}+\frac{5}{2}$.

For the $W=3$ case, the Hamiltonian is

$$
H_{3}\left(k_{x}\right)=\left[\begin{array}{ccc}
h_{1}\left(k_{x}\right) & h_{2} & 0 \\
h_{2}^{\dagger} & h_{1}\left(k_{x}\right) & h_{2} \\
0 & h_{2}^{\dagger} & h_{1}\left(k_{x}\right)
\end{array}\right] .
$$

The lower boundary is described by $\mu / V=-2.76 t / V$. 

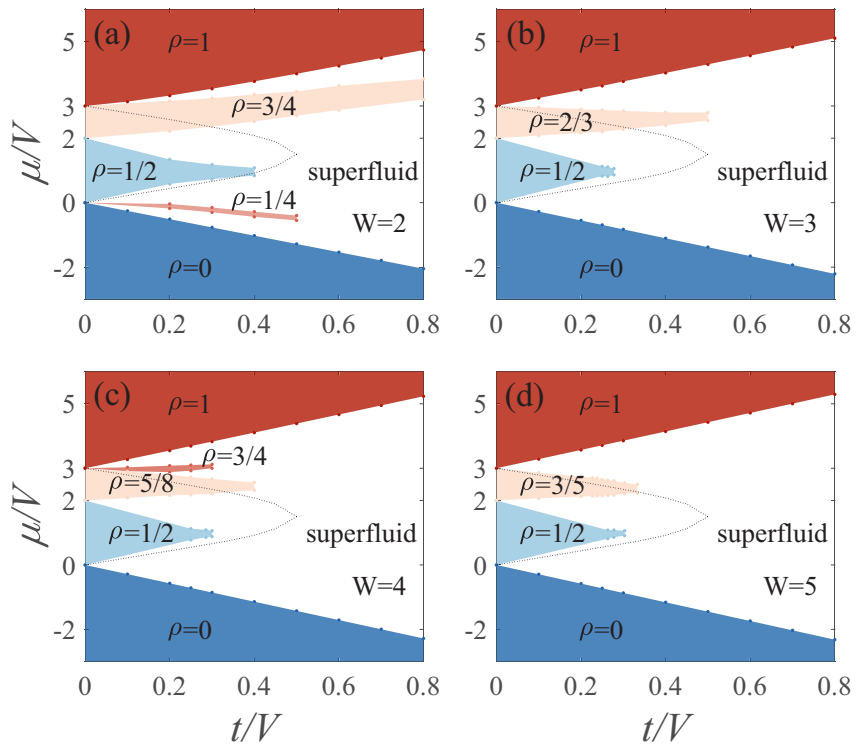

FIG. 3. Phase diagrams of $W=2,3,4,5$ honeycomb lattice ribbons contain superfluid regions and insulator phases at specific fillings. Dotted lines enclose the $\rho=\frac{1}{2} \mathrm{CDW}$ insulator of hard-core bosons on a periodic two-dimensional honeycomb lattice [16-18]. Ribbon length $L=24$.

Finally, for $W=4$,

$$
H_{4}\left(k_{x}\right)=\left[\begin{array}{cccc}
h_{1}\left(k_{x}\right) & h_{2} & 0 & 0 \\
h_{2}^{\dagger} & h_{1}\left(k_{x}\right) & h_{2} & 0 \\
0 & h_{2}^{\dagger} & h_{1}\left(k_{x}\right) & h_{2} \\
0 & 0 & h_{2}^{\dagger} & h_{1}\left(k_{x}\right)
\end{array}\right] .
$$

From the eigenvalue of the band bottom, we have $\mu / V=$ $-2.85 t / V$ describing the phase boundary for $\rho=0$. The phase boundary for $\rho=1$ can be obtained numerically in the hole representation. Figure 2 shows the bands for $W=2-5$, and Fig. 3 gives the corresponding phase diagrams, which are further discussed in the following section.

The band structure of the honeycomb lattice consists of two inequivalent Dirac points, which are characterized by $\pm \pi$ Berry phases [19]. As a result of the nontrivial topological property, localized flat bands connecting the two Dirac points appear on the zigzag edges [20-23]. The development of the flat bands is already evident in the full band structure of Fig. 2. However, Fig. 4 makes this more clear by focusing on the low-energy bands only and including data for $W=8$. Figure 4 emphasizes that as the widths $W$ of the ribbons increase, the lengths of the flat bands grow. As $W \rightarrow \infty$, the full flat band along the edges is recovered. The band bottom corresponds to the chemical potential at which the hard-core bosons begin to fill into the system, which determines the phase boundary for $\rho=0$.

\section{PHASE DIAGRAM}

Phase diagrams of ribbons with $W=2,3,4,5$ are shown in Fig. 3. In the atomic limit $(t / V=0)$, the density abruptly jumps from empty $(\rho=0)$ to half filled $\left(\rho=\frac{1}{2}\right)$ at $\mu / V=0$, with bosons placed so they never occupy adjacent sites.
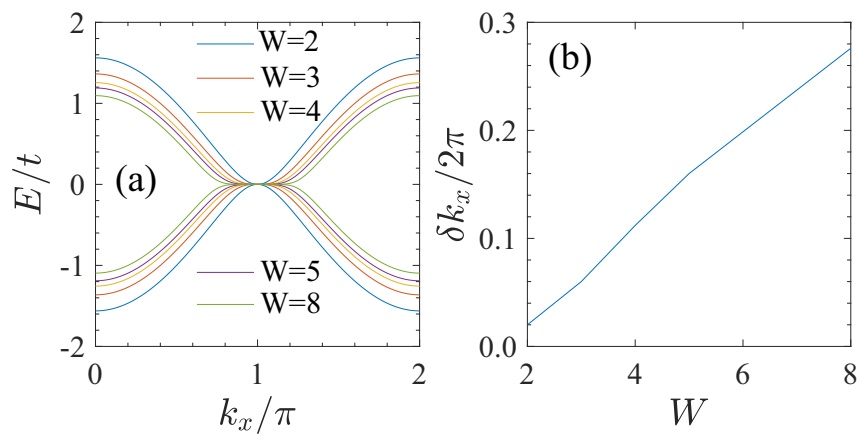

FIG. 4. Low-energy bands of ribbons with several $W$ and $L=$ $\infty$. Inset shows the ratio of the range $\delta k_{x}$ of the flat band to the full one-dimensional Brillouin zone, as a function of $W . \delta k_{x} / 2 \pi \sim 1 / 3$ for large $W$.

At $\rho=\frac{1}{2}$, no further bosons can be added without being neighbors, costing energy $\propto V$ : there is a jump in $\mu$. If the half-filled bosons are placed so that they occupy only a single sublattice, the empty sites of one of the boundaries are special: they interact with only two neighboring occupied sites. Thus the $\rho=\frac{1}{2} \mathrm{CDW}$ insulator terminates at $\mu / V=2$ [24]. Once these special sites are completely occupied, the increase in density pauses again until $\mu / V=3$, at which point bosons are added to the remaining empty sites with three occupied neighbors, completely filling the lattice. This atomic-limit picture explains the positions of the insulating lobe bases in Fig. 3.

The $\rho=\frac{1}{2}$ insulator for $0<\mu / V<2$ has a $(W+1)$-fold degeneracy, including two single-sublattice CDWs and ( $W-$ 1) configurations with a domain wall arranged along each row of vertical bonds. As noted earlier, the key observation for the presence of self-organized domain walls of Fig. 1 is that a domain wall has a pair of "edges" where empty sites have only two occupied neighbors. This greater multiplicity of special sites compared to a single sublattice leads to a lower energy at finite hopping $t / V \neq 0$ : the second-order energy decrease when a boson hops onto a special site is $-t^{2} / V$ compared to the $-t^{2} / 2 V$ for hopping within the CDW. Figure 1 illustrates these different hopping processes and the larger overall energy decrease, $-5 t^{2} / 2 \mathrm{~V}$, of a site adjacent to a domain wall, compared to $-3 t^{2} / 2 \mathrm{~V}$ gained by a boson inside the CDW. Filling half of the special sites in the domain-wall phase also explains the densities $\rho=\frac{1}{2}+\frac{1}{2 W}$ of the $2<\mu / V<3$ insulating lobes of Fig. 3.

The atomic insulator phases initially persist at small $t / V$, but the range in chemical potential over which they are stable decreases. They completely disappear beyond a critical value of $t / V$. At nonzero $t / V$, all these insulators are separated by incommensurate superfluid regions.

Quantum phases suggested by these strong-coupling arguments can be precisely determined using the SSE by measuring the average density $\rho=\frac{1}{N} \sum_{i}\left\langle n_{i}\right\rangle$ and the superfluid density $\rho_{s}=\left\langle W^{2}\right\rangle / 4 \beta t$, where $W$ is the winding number counting the net number of times the paths of the particles have wound around the periodic cell $[25,26]$, and $\beta$ is the inverse temperature. Insulating behavior is characterized by $\rho_{s}=0$ and a plateau of $\rho$ representing the persistence of 


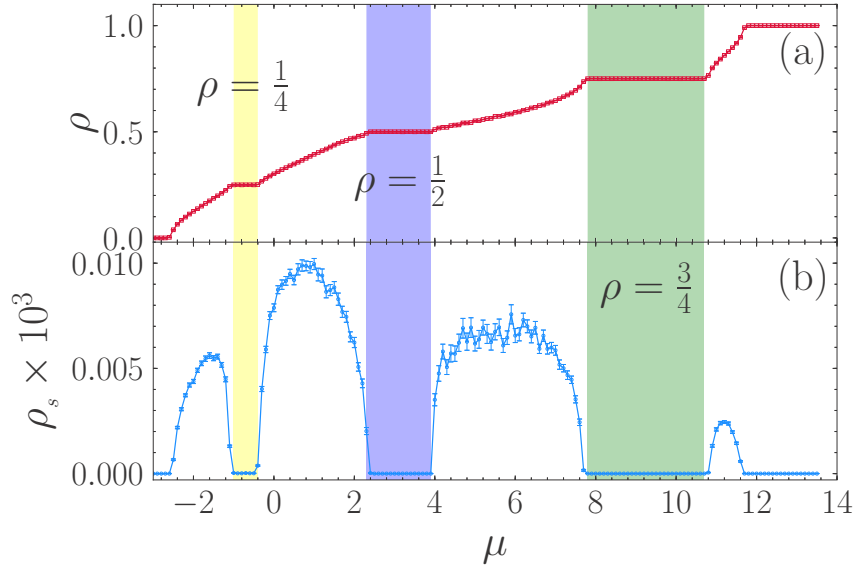

FIG. 5. The average density (a) and superfluid density (b) as a function of $\mu$ at $t / V=0.3$ on a $W=2$ and $L=24$ ribbon.

the atomic-limit steps in the chemical potential to finite $t / V$. Conversely, the superfluid has nonzero $\rho_{s}$ and finite compressibility $\kappa=\partial \rho / \partial \mu$. These features are clearly seen in the SSE results of Fig. 5 for $W=2$. A collection of plots like Fig. 5 for different $t / V$ generates the phase diagram in Fig. 3.

Compared to that of the periodic honeycomb lattice, the regions of the $\rho=\frac{1}{2}$ insulators of Fig. 3 are greatly reduced, and there appear new insulating regions at the filling $\rho=\frac{1}{2}+$ $\frac{1}{2 W}$. There is an additional valence-bond insulator at $\rho=\frac{1}{4}\left(\frac{3}{4}\right)$ for $W=2$ (4) which has no atomic counterpart [27,28].

\section{STRONG COUPLING}

The ED and DMRG methods can be used to obtain further details of the evolution of the low-energy spectrum and site densities away from the atomic $(t=0)$ limit. The $(W+1)$ fold degeneracy of the atomic limit $\rho=\frac{1}{2}$ insulator is lifted by the hopping. For the $W=2$ case, the ground state is the unique domain-wall phase. The first- and second- excited

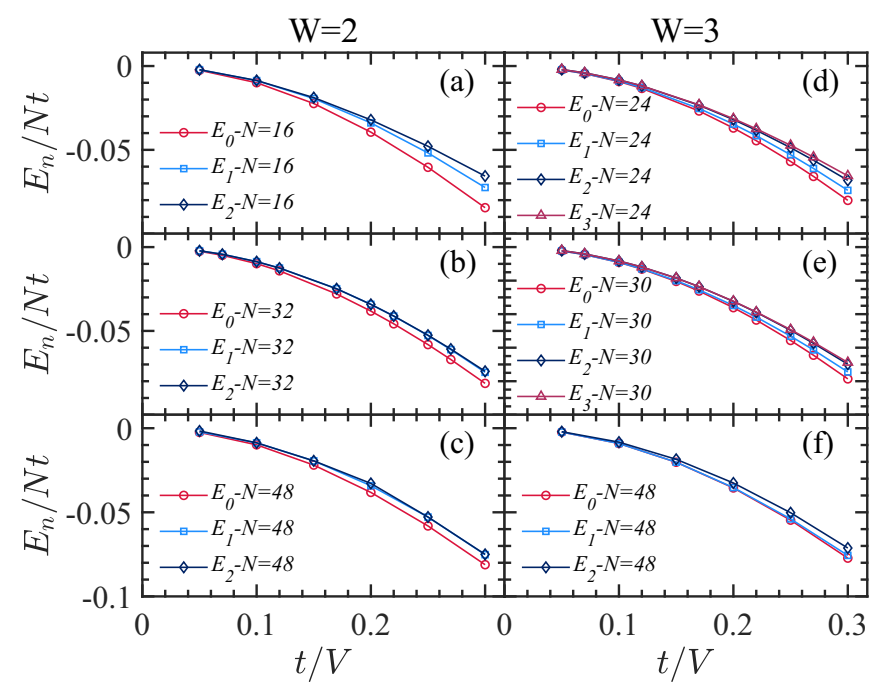

FIG. 6. Several of the lowest eigenenergies calculated by the ED method: (a) $W=2, L=4$; (b) $W=2, L=8$; (d) $W=3, L=4$; (e) $W=3, L=5$. The three lowest eigenenergies from the DMRG methods on $N=48$ sites: (c) $W=2$, (f) $W=3$.

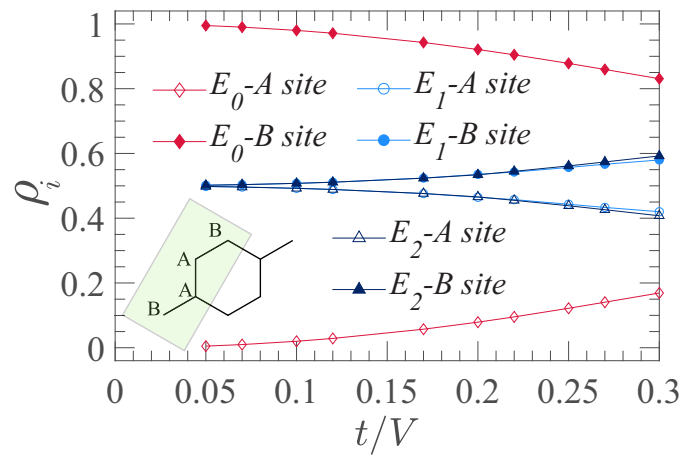

FIG. 7. The profiles of the local densities in the ground, first-, and second-excited states. The first- and second-excited states are linear combinations of the two single-sublattice CDW states. The lattice size is $W=2$ and $L=8$. Inset shows the unit cell of the $W=2$ ribbon and equivalent sites, due to inversion point group symmetry.

states are linear combinations of the single-sublattice CDW states to preserve the symmetries of the Hamiltonian. As shown for $W=2$ in Figs. 6(a)-6(c), as $t / V$ increases, $W+$ $1=3$ distinct eigenenergy curves emerge from the degenerate $t / V=0$ limit. The $(N=16)$-site ED eigenenergies of the two mixed single-sublattice CDW states lie above the domain-wall state and are slightly split by finite-size effects. This finite-size splitting is significantly reduced in the $(N=32)$-site ED and the $(N=48)$-site DMRG calculations.

The site densities and the density-density correlations in Figs. 7 and 8(a) confirm that the lowest eigenenergy is associated to the unique domain-wall phase. When $t$ is nonzero, the single-sublattice CDW states form linear combinations and in the resulting state superpositions, all sites have average filling around 0.5. However the domain-wall state, because of inversion symmetry, has two inequivalent sets of sites, half of which have densities which are low, and half which are high (see Fig. 7). In the domain-wall phase, both edge sites of the unit cell have high densities, while one of them is occupied and the other is not in the CDW phase. The density-density correlations between such sites have distinct values, which are large for the domain-wall phase and small for the CDW [see Fig. 8(a)]. Thus the presence of two wellseparated $\rho_{i}(t / V)$ trajectories and the large density-density correlation between opposite-edge sites are "smoking guns"

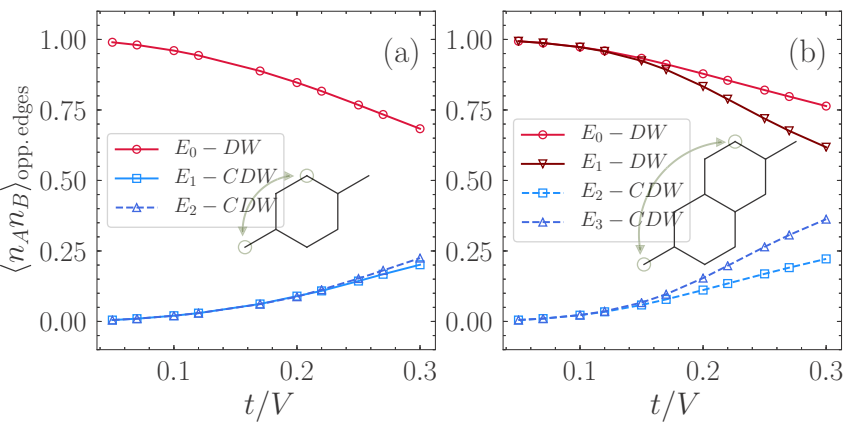

FIG. 8. The density-density correlations between the oppositeedge sites of the unit cell for several of the lowest eigenenergies. The lattice sizes are (a) $W=2$ and $L=8$, (b) $W=3$ and $L=5$. 

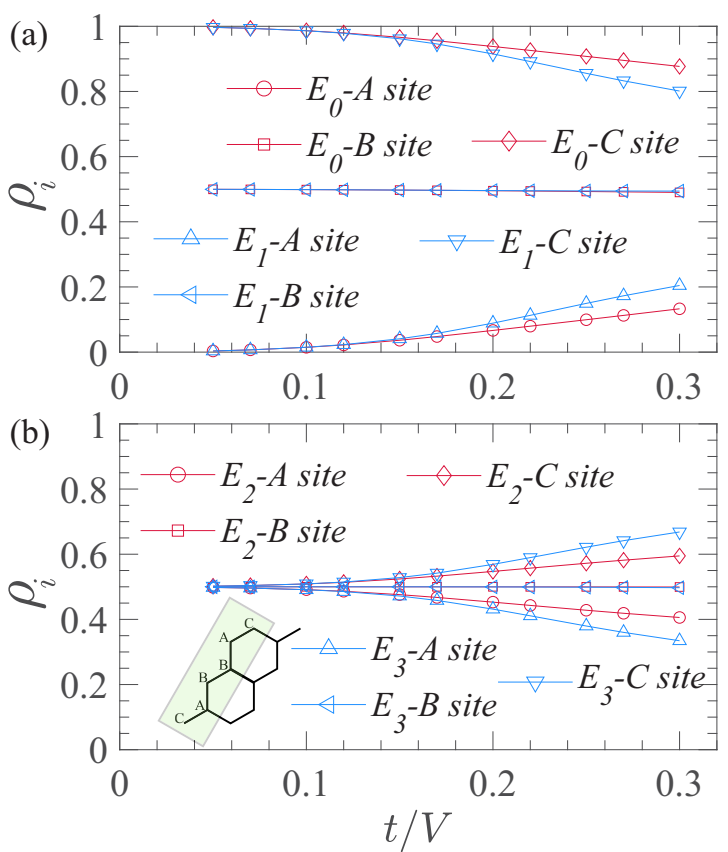

FIG. 9. (a) The profiles of the local densities in the ground- and first-excited states, which are linear combinations of the two domainwall phases. (b) The profiles of the local densities in the secondand third-excited states, which are linear combinations of the two single-sublattice CDW states. The lattice size is $W=3$ and $L=5$.

that the ground state has a domain wall, as already suggested by the strong-coupling argument.

We also demonstrate the existence of a domain wall in the $W=3$ ground state. In Figs. $6(\mathrm{~d})-6(\mathrm{f})$, the $(W+1=4)$ fold atomic-limit degeneracy is lifted by hopping. The lowest four eigenenergies are grouped into two sets, each of which contains two mixed domain-wall or CDW states. Due to the finite-size effect, the degeneracy of each set is split. In the larger lattice results, the two lowest states become nearly degenerate, and so are the two upper ones.

If one averages the $\rho_{i}$ of the two domain-wall states, one finds two "occupied" sites which have $\rho_{i}$ large in both states, two "empty" sites which have $\rho_{i}$ small in both states, and two sites which exchange $\rho_{i}$ small and large. This leads to three $\rho_{i}$ trajectories: large, small, and approximately half filled. Meanwhile, each of the CDW states has six inequivalent sites, three high and three low density, which all exchange between the two degenerate cases. When averaged, all sites would therefore have $\rho_{i} \sim 0.5$. The site densities of the lower and upper two states are plotted in Fig. 9 and are consistent with those of the domain-wall and CDW phases. The density-density correlation between opposite-edge sites in the domain-wall phase is also much larger than that in the CDW phase [see Fig. 8(b)]. Thus the local densities and densitydensity correlations observed for $W=3$ offer compelling evidence that the ground state manifests a charge domain wall.

\section{THE MAGNON BANDS AND BERRY CURVATURE}

Having established the phase diagram and the existence of domain walls in the $\rho=1 / 2$ insulator, we now focus on behavior at the interface. The topological property of the bulk
CDW phase of hard-core bosons is first investigated. The Bose-Hubbard Hamiltonian in Eq. (1) is equivalent to a spin$1 / 2 X X Z$ model through a mapping $S_{i}^{+}=b_{i}^{\dagger}$ and $S_{i}^{z}=n_{i}-\frac{1}{2}$,

$$
\begin{aligned}
H= & -t \sum_{\langle i, j\rangle}\left(S_{i}^{+} S_{j}^{-}+S_{i}^{-} S_{j}^{+}\right) \\
& +V \sum_{\langle i, j\rangle}\left(S_{i}^{z}+\frac{1}{2}\right)\left(S_{j}^{z}+\frac{1}{2}\right)-\mu \sum_{i}\left(S_{i}^{z}+\frac{1}{2}\right) .
\end{aligned}
$$

Using the Holstein-Primakoff transformation, the spin operators are expressed in terms of bosonic creation and annihilation operators. The honeycomb lattice is bipartite; thus the transformation on sublattice $A$ is defined as

$$
\begin{aligned}
& S_{A, i}^{+}=\left(\sqrt{2 S-a_{i, A}^{\dagger} a_{i, A}}\right) a_{i, A}, \\
& S_{A, i}^{-}=a_{i, A}^{\dagger}\left(\sqrt{2 S-a_{i, A}^{\dagger} a_{i, A}}\right), \\
& S_{A, i}^{z}=S-a_{i, A}^{\dagger} a_{i, A} .
\end{aligned}
$$

Conversely, on sublattice $B$, the spin is in the opposite direction for antiferromagnetic order. Thus the spin operators are defined as

$$
\begin{aligned}
& S_{B, i}^{+}=a_{i, B}^{\dagger}\left(\sqrt{2 S-a_{i, B}^{\dagger} a_{i, B}}\right), \\
& S_{B, i}^{-}=\left(\sqrt{2 S-a_{i, B}^{\dagger} a_{i, B}}\right) a_{i, B}, \\
& S_{B, i}^{z}=a_{i, B}^{\dagger} a_{i, B}-S .
\end{aligned}
$$

Expanding the square root in Eqs. (9) and (10) in powers of $1 / S$, the zeroth-order terms are kept in the linear spin-wave theory. Then the bosonic tight-binding Hamiltonian becomes

$$
\begin{aligned}
H= & -t \sum_{\langle i, j\rangle}\left(a_{i, A} a_{j, B}+a_{i, A}^{\dagger} a_{j, B}^{\dagger}\right) \\
& +V \sum_{\langle i, j\rangle}\left(1-a_{i, A}^{\dagger} a_{i, A}\right) a_{i, B}^{\dagger} a_{i, B} \\
& -\mu \sum_{i \in A}\left(1-a_{i, A}^{\dagger} a_{i, A}\right)-\mu \sum_{i \in B} a_{i, B}^{\dagger} a_{i, B},
\end{aligned}
$$

where $a_{i, \alpha}^{\dagger}, a_{i, \alpha}(\alpha=A, B$ denoting the sublattice) are the bosonic creation and annihilation operators [29]. In momentum space, $H=\sum_{\mathbf{k}} \psi_{\mathbf{k}}^{\dagger} \mathcal{H}(\mathbf{k}) \psi_{\mathbf{k}}$, where $\psi_{\mathbf{k}}=\left\{a_{A, \mathbf{k}}, a_{B,-\mathbf{k}}^{\dagger}\right\}^{T}$, and

$$
\mathcal{H}(\mathbf{k})=\left[\begin{array}{cc}
\mu & f(\mathbf{k}) \\
f^{*}(\mathbf{k}) & 3 V-\mu
\end{array}\right],
$$

with $f(\mathbf{k})=-t\left(1+e^{-i \mathbf{k} \cdot \mathbf{a}_{1}}+e^{-i \mathbf{k} \cdot \mathbf{a}_{2}}\right) \quad\left[\mathbf{a}_{1}=(\sqrt{3}, 0), \mathbf{a}_{2}=\right.$ $(\sqrt{3} / 2,3 / 2)$ are the primitive vectors]. To diagonalize the above Hamiltonian, we consider the following non-Hermitian matrix

$$
\sigma_{z} \mathcal{H}(\mathbf{k})=\left[\begin{array}{cc}
\mu & f(\mathbf{k}) \\
-f^{*}(\mathbf{k}) & -3 V+\mu
\end{array}\right]
$$



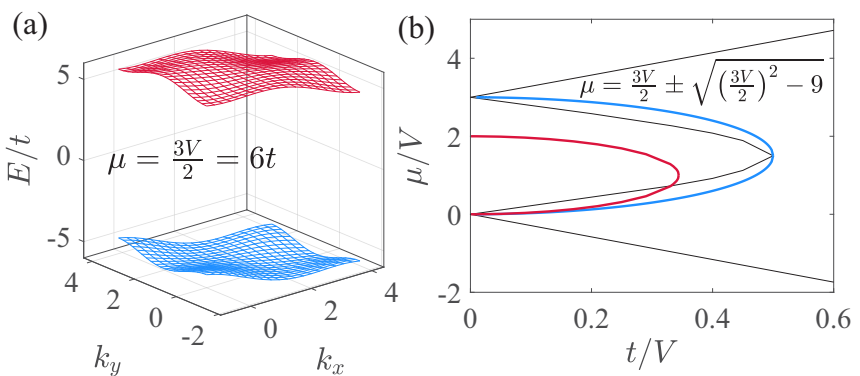

FIG. 10. (a) The magnon band structure. The parameters are $\mu=\frac{3 V}{2}=6 t$, when $E^{+}, E^{-}$are identical and we plot $-E^{-}$to display this. (b) The phase diagram of the Bose-Hubbard model on the periodic honeycomb lattice and zigzag ribbon. The black (blue) curves are the phase boundaries from the QMC method (the spinwave approximation) on the periodic honeycomb lattice. The red curves are from the spin-wave approximation for a zigzag ribbon with the width $W=12$.

The eigenvalues are given by $E_{\mathbf{k}}^{ \pm}=\mu-\frac{3 V}{2} \pm \epsilon(\mathbf{k})$ with $\epsilon(\mathbf{k})=\sqrt{\left(\frac{3 V}{2}\right)^{2}-|f(\mathbf{k})|^{2}}$. The eigenvector matrix is

$$
\mathcal{U}_{\mathbf{k}}=\left[\begin{array}{cc}
\cosh \theta_{\mathbf{k}} e^{i \phi_{\mathbf{k}}} & -\sinh \theta_{\mathbf{k}} \\
-\sinh \theta_{\mathbf{k}} & \cosh \theta_{\mathbf{k}} e^{-i \phi_{\mathbf{k}}}
\end{array}\right],
$$

where $\sinh 2 \theta_{\mathbf{k}}=\frac{|f(\mathbf{k})|}{\epsilon(\mathbf{k})}, \tan \phi_{\mathbf{k}}=\frac{\operatorname{Im} f(\mathbf{k})}{\operatorname{Re} f(\mathbf{k})}$. The first (second) column is the eigenvector $u_{+, \mathbf{k}}\left(u_{-, \mathbf{k}}\right)$ corresponding to $E_{\mathbf{k}}^{+}$ $\left(E_{\mathbf{k}}^{-}\right)$. The Hamiltonian is thus diagonalized by the transformation $\mathcal{U}_{\mathbf{k}}^{\dagger} \mathcal{H}(\mathbf{k}) \mathcal{U}_{\mathbf{k}}=\operatorname{diag}\left(E_{\mathbf{k}}^{+},-E_{\mathbf{k}}^{-}\right)$.

Thus the magnon band structure, i.e., the excitation of the mapped spin-1/2 $X X Z$ model, has two branches: $E_{\mathbf{k}}^{ \pm}= \pm\left(\mu-\frac{3 V}{2}\right)+\epsilon(\mathbf{k})$, where $\epsilon(\mathbf{k})=\sqrt{\left(\frac{3 V}{2}\right)^{2}-|f(\mathbf{k})|^{2}}$. In Fig. 10(a), we plot the magnon band structure at $\mu=\frac{3 \mathrm{~V}}{2}=$ $6 t$, which consists of two gapped branches.

The above magnon bands of the mapped spin-1/2 XXZ model correspond to the excitation spectrum above the CDW insulator of the Bose-Hubbard model, which is the superfluid. When the spectrum becomes gapless, i.e., $E_{\mathbf{k}}^{ \pm}=0$, superfluid begins to appear in the system. Thus $E_{\mathbf{k}}^{ \pm}=0$ determine the phase boundary between the CDW and superfluid phases, which is $\mu=\frac{3 V}{2}-\sqrt{\left(\frac{3 V}{2}\right)^{2}-(3 t)^{2}}$. We plot the phase boundary from the spin-wave approximation in Fig. 10(b). It is qualitatively consistent with the exact phase diagram from the QMC method except that the range in the chemical potential is slightly increased. We also determine the phase boundary of the $\rho=\frac{1}{2}$ domain-wall phase on a $W=12$ zigzag ribbon, which greatly shrinks from that of a periodic system.

The Berry curvature associated with each magnon band is given by

$$
\Omega_{\lambda}(\mathbf{k})=\frac{\partial A_{y}(\mathbf{k})}{\partial k_{x}}-\frac{\partial A_{x}(\mathbf{k})}{\partial k_{y}},
$$

where $A_{i}=-i\left\langle u_{\lambda, \mathbf{k}}\left|\frac{\partial}{\partial k_{i}}\right| u_{\lambda, \mathbf{k}}\right\rangle(i=x, y)$ is the Berry potential. $\lambda= \pm$ denotes the two magnon bands [30-32]. The Berry curvature is peaked at the Brillouin zone (BZ) corners, Fig. 11(a), and is antisymmetric with respect to the inversion center $\mathbf{k}=(0,0)$. The sum of the Berry curvature of each band in
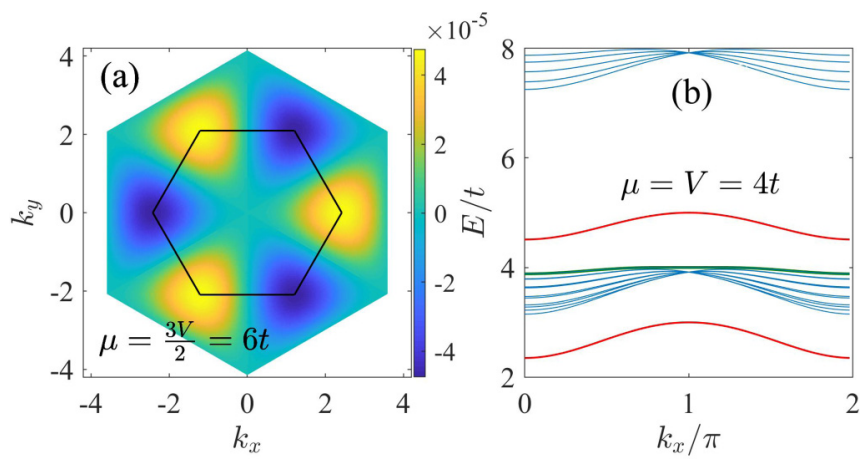

FIG. 11. (a) The Berry curvature associated with the upper magnon band, which differs from that of the lower band by a sign. The first Brillouin zone is marked by black lines. The parameters are $\mu=\frac{3 V}{2}=6 t$. (b) The excitation spectrum on a $W=12$ ribbon with a zigzag domain wall in the middle. The red curves represent states localized near the domain wall. The green curves are twofold degenerate, and are associated with the zigzag edges. The parameters are $\mu=V=4 t$.

the BZ (the Chern number) vanishes identically. The Berry curvatures for the two $\rho=\frac{1}{2} \mathrm{CDW}$ insulators differ by an overall sign; thus the Berry curvature changes the sign across the domain wall. Due to bulk-boundary correspondence, there should appear in-gap domain-wall states $[33,34]$. As shown in Fig. 11(b), there are two such branches associated with the domain wall. One of them is at the bottom of the magnon excitation spectrum, and it corresponds to the superfluid above the $\rho=\frac{1}{2} \mathrm{CDW}$ insulator, which is localized near the domain wall.

\section{THE DOMAIN-WALL SUPERFLUID}

For the density $\rho=\frac{1}{2}$, the domain wall is thus formed by a quasi-1D region with negligible occupancy, confined by robust CDW phases. As the chemical potential is further increased, bosons are preferentially added to the empty sites forming the domain wall, since they only interact with two occupied neighbors in contrast with three neighbors of an empty CDW site. A single extra boson can hop freely along the chain, lowering its kinetic energy without changing the interacting energy. Additional added particles behave effectively as interacting bosons in $1 \mathrm{D}$, which condense to superfluid transport down the domain wall. The values of the superfluid density, Fig. 5(b), follow a dome shape, and are maximal at density $\sim \frac{1}{2}+\frac{1}{4 W}$ when half of such empty sites are occupied. After the domain wall is full, the superfluid vanishes, and the system becomes a $\rho=\frac{1}{2}+\frac{1}{2 W}$ insulator. Figure 12 shows the density for $W=2,4$. The presence of two sets of wellseparated local traces is consistent with the $\rho=\frac{1}{2}$ insulator being a domain-wall phase (see discussion of Fig. 7).

While the local density of the sites on the domain wall with small occupation increases, that of the high-occupancy sites first decreases even as $\mu$ grows. This anomalous behavior is a signature of the flow of bosons onto the domain wall and the appearance of a superfluid localized near the domain wall. It is noteworthy that the domain-wall superfluid coexists with diagonal (density) order: the zigzag honeycomb nanoribbon realizes an exotic one-dimensional supersolid [35,36]. The 


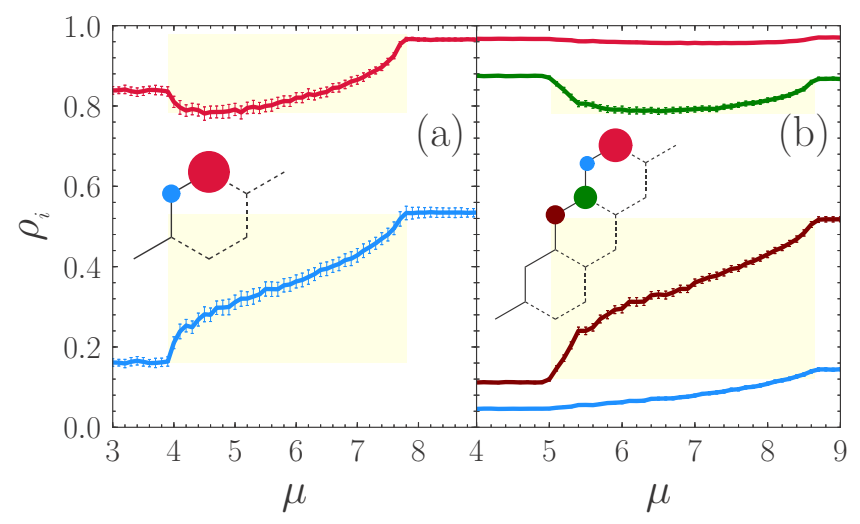

FIG. 12. The local densities as a function of the chemical potential: (a) $W=2$ and $t / V=0.3$, (b) $W=4$ and $t / V=0.25$. Due to the geometric symmetries, only nonequivalent sites are shown. Here the length of the ribbons is $L=24$.

emergence of this superfluid is a manifestation of change in topological properties when crossing the domain wall.

To verify the localization of the superfluid near the domain wall, we show the single-particle correlator $\left\langle b_{0}^{\dagger} b_{r}\right\rangle$ in Fig. 13. The correlator along the zigzag chain on the domain wall is slower than a power-law decay with distance, which is characteristic of a gapless quasi-1D superfluid. In contrast, the excitation is gapped for the $\rho=\frac{1}{2}$ domain-wall insulator, and the correlator decays exponentially. As one moves away from the domain wall, the correlator becomes increasingly shortranged, and $\rho_{s}$ decreases. For wide ribbons, the superfluid

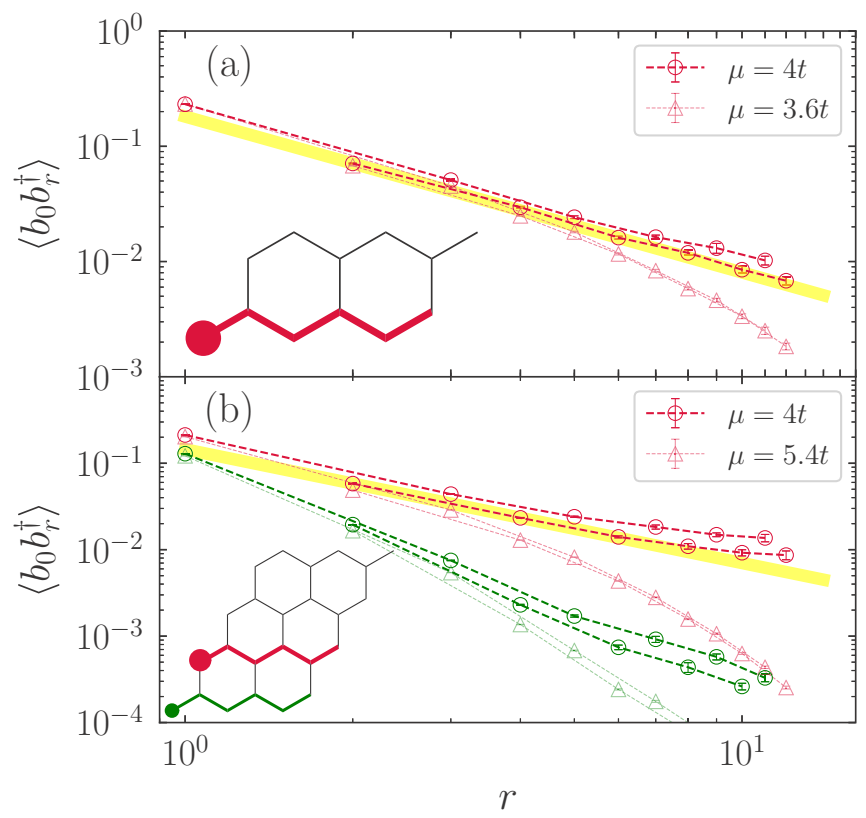

FIG. 13. The single-particle correlator $\left\langle b_{0}^{\dagger} b_{r}\right\rangle$ : (a) $W=2$, (b) $W=4$. The circle symbols on the inset geometries mark the reference site $r=0$. The correlators are calculated along the thick zigzag lines, and only nonequivalent lines are shown. The thick yellow lines are plotted as guides to algebraic behavior. Up triangles connected by dotted lines refer to the insulating regime with $\rho=\frac{1}{2}$. Parameters are the same as those in Fig. 5. density decays exponentially with the distance away from the domain wall.

\section{CONCLUSIONS}

We studied hard-core bosons on zigzag edge honeycomb lattice ribbons using exact simulations. The phase diagram contains superfluid and insulating phases and, remarkably, at $\rho=\frac{1}{2}$ filling the ground state contains a charge domain wall rather than occupation of a single sublattice. This "selforganized domain wall" separates CDW regions with opposite Berry curvature, and supports superfluid transport in coexistence with diagonal (density) order. Our results demonstrate that honeycomb ribbons provide a concrete geometry for the observation of bosonic topological phenomena.

This physics can be explored experimentally. Cold atoms in optical lattices provide a well-established means to emulate the Bose-Hubbard model [37]; large values of $U$ which achieve the hard-core limit can be attained, and the honeycomb geometry has been generated [38-40]. The use of a synthetic dimension may also provide a simple realization of honeycomb ribbons [41,42]. New tools based on quantum gas microscopes allow the observation of the density profile at the level of individual atoms [43-46] and hence direct comparison with our real-space measurements. The Berry curvature can also be obtained via interferometric techniques $[47,48]$.

\section{ACKNOWLEDGMENTS}

The authors thank G. Batrouni, G. Chen, and F. Nori for helpful discussions. H.G. acknowledges support from NSFC Grant No. 11774019. X.Z. and S.F. are supported by the National Key Research and Development Program of China under Grant No. 2016YFA0300304, and NSFC under Grants No. 11574032 and No. 11734002. R.M. acknowledges support from NSAF-U1930402, and from NSFC Grants No. 11674021, No. 11851110757, and No. 11974039. R.T.S. was supported by DOE Grant No. DE-SC0014671.

\section{APPENDIX A: THE DOMAIN-WALL SUPERFLUID ON A WIDER RIBBON}

To characterize the domain-wall superfluid more completely, and to show its nature on wider ribbons, we perform QMC simulations on a $W=12$ and $L=24$ ribbon. Since there are more approximately degenerate $\rho=\frac{1}{2}$ phases on wider ribbons, a small pinning field is used to select a specific configuration [49]. The pinning field is a staggered potential with the value $-|\Delta|(|\Delta|)$ on each occupied (unoccupied) site of the targeted domain-wall phase. In the following simulations, the strength of the pinning field is set to $|\Delta|=0.1$, and we focus on the phase with the domain wall in the middle.

Figure 14 shows the average density and superfluid density as a function of chemical potential at $V / t=4$. As the chemical potential increases, bosons are continuously added to the $\rho=\frac{1}{2}$ domain-wall phase until the domain wall is full, and the ribbon becomes a $\rho=\frac{1}{2}+\frac{1}{2 W}$ insulator. Between the two insulators, the superfluid density is nonzero, implying the system is a superfluid. 


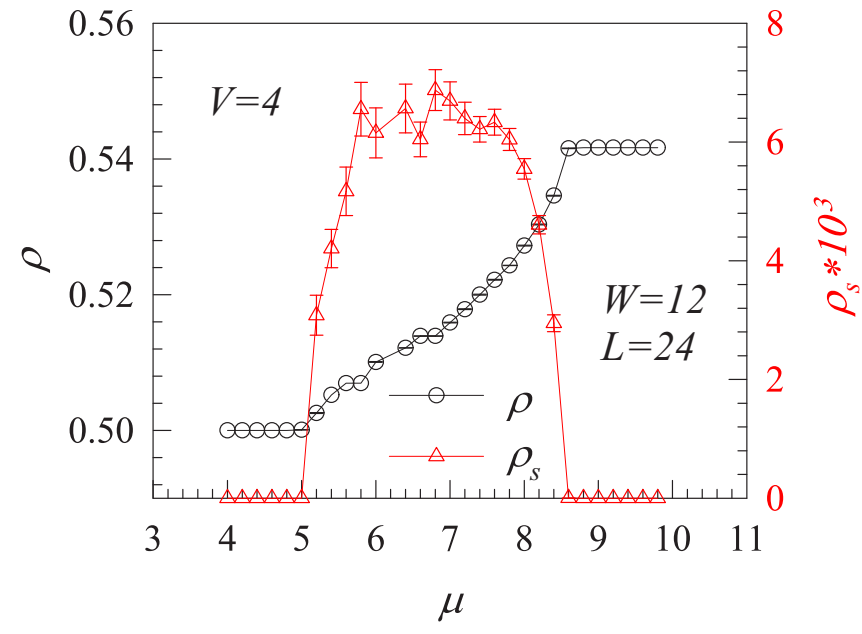

FIG. 14. The average density and superfluid density as a function of $\mu$ at $V / t=4$ on a $W=12$ and $L=24$ ribbon.

This wide $W=12$ ribbon allows for a more detailed examination of superfluid localization near the domain wall, and the formation of a one-dimensional superfluid channel. Figure 15 shows the local densities as functions of the chemical potential. For the $\rho=\frac{1}{2}$ insulator, the profile of the local density indicates it is a domain-wall phase with the domain wall in the middle of the ribbon. From about $\mu=5$, additional bosons are added to the system. The value on the low-occupation sites of the domain wall increases significantly. In the $\rho=$ $\frac{1}{2}+\frac{1}{2 W}$ insulator, the site densities approach $\rho_{i} \sim 0.5$, which corresponds to the case in which the domain wall is full. The local densities are only slightly affected for sites more than $2 a$ ( $a$ the lattice constant) away from the domain wall. Thus the added bosons mainly reside near the domain wall. It is also noted that the local densities on the highly occupied sites near the domain wall first decrease. This implies the bosons

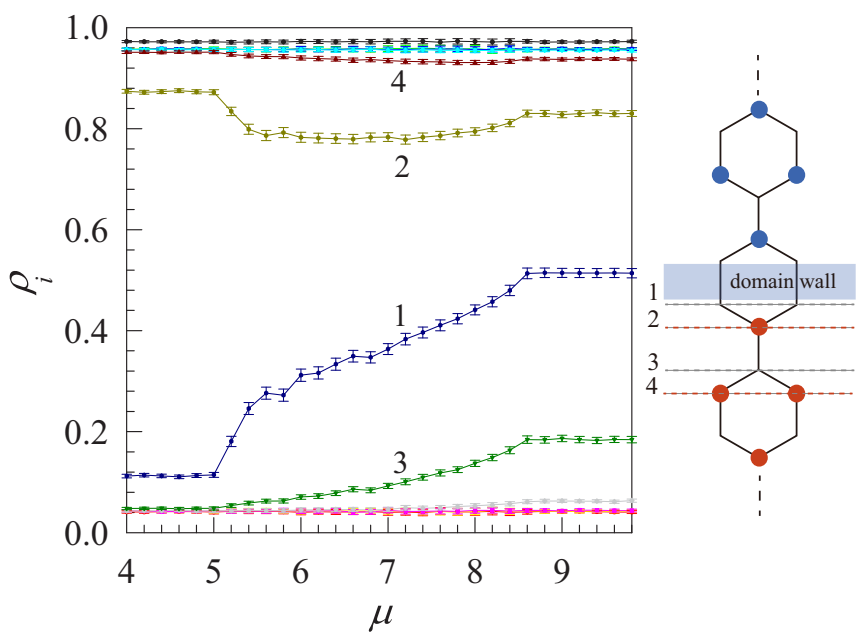

FIG. 15. The local densities as functions of chemical potential. Due to the translation symmetry, the sites of each sublattice on the lines parallel to the domain wall are equivalent (see the right panel). In addition, the system is symmetric about the domain wall. So we only show the values on nonequivalent sites, which are from the upper (lower) half unit cell. The parameters are the same as those in Fig. 14.

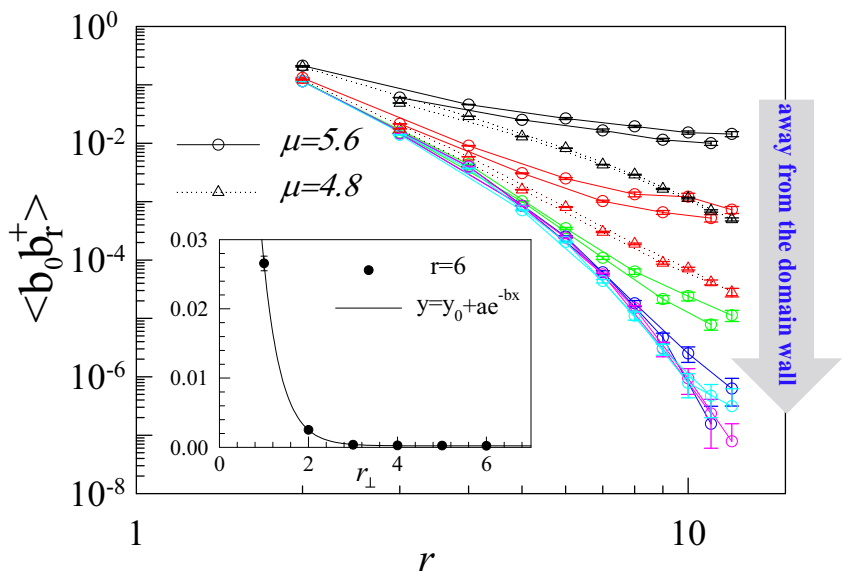

FIG. 16. The single-particle correlator $\left\langle b_{0}^{\dagger} b_{r}\right\rangle$ as a function of distance from the reference point. Due to the symmetries, only nonequivalent zigzag lines are shown. For comparison, we also calculate $\left\langle b_{0}^{\dagger} b_{r}\right\rangle$ in the $\rho=\frac{1}{2} \mathrm{CDW}$ phase, and show the results along two zigzag chains closest to the domain wall. Inset shows $\left\langle b_{0}^{\dagger} b_{r}\right\rangle$ as a function of distance $r_{\perp}$ away from the domain wall at fixed $r=6$. The data are well fitted by an exponential decay. Here the parameters are the same as those in Fig. 14.

begin to flow between high- and low-occupation sites, which is consistent with the appearance of superfluid. Since the decrease is most significant on the domain wall, the gapless superfluid is mainly around it.

We can also calculate the single-particle correlator for this wider $W=12$ ribbon to get a more detailed picture of the physics as the distance $r_{\perp}$ away from the domain wall increases. As for smaller $W$, the decay of the correlator becomes rapid with larger $r_{\perp}$, suggesting that the superfluid density decreases (see Fig. 16). For the zigzag chain on the domain wall, the decay of the correlator is slower than power law. However the decay becomes exponential from the fourth zigzag chain, where the superfluid begins to vanish. Moreover the curves remain almost unchanged for the far zigzag chains, manifesting the uniform bulk CDW order there. The inset shows $\left\langle b_{0}^{\dagger} b_{r}\right\rangle$ as a function of $r_{\perp}$ (the distance away from the domain wall) at fixed $r=6$. The data are well fitted using an exponential decay. Thus the superfluid is localized near the domain wall, and decays exponentially into the bulk.

\section{APPENDIX B: THE DOMAIN WALL WITH TURNS}

It is interesting to see whether it is possible to have domain walls with turns. The domain wall organized by the NN interactions should be straight and along the ribbon. The open zigzag edges of the ribbons are created by removing a line of vertical bonds. Then both sites connected by such a bond can be occupied. To be at half filling, we have a line of vertical bonds empty in the bulk, which form the "self-organized" domain wall. Once the domain wall has turns, nonvertical bonds crossing should be empty. To maintain the number of bosons at half filling, there must appear adjacent occupied sites connected by periodic boundary conditions, which induce interacting energy. As shown in Fig. 17(a), a Z-shaped domain wall crosses one nonvertical bond, and there appears a 


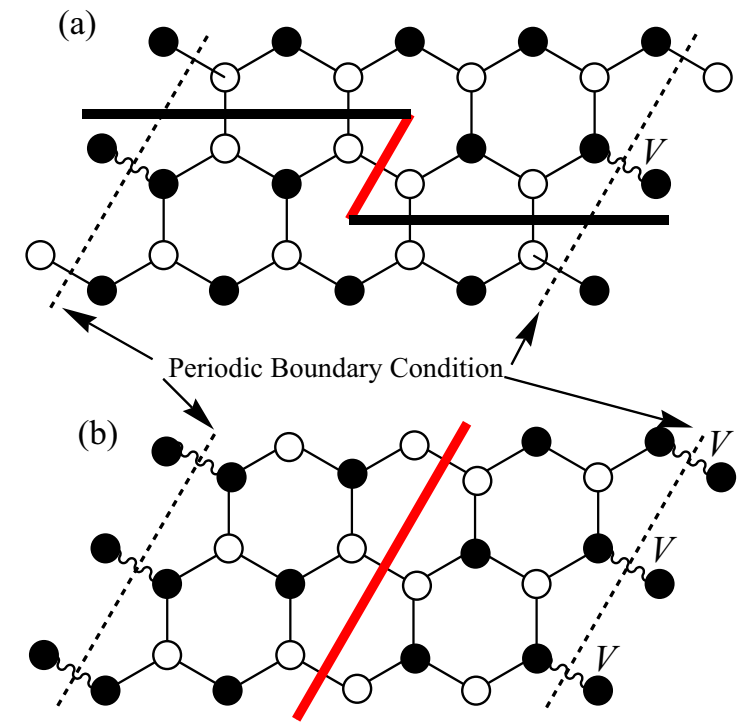

FIG. 17. Schematic demonstrations of (a) a domain wall with two turns, (b) a domain wall along a direction other than the longer axis of the ribbon. Both configurations are not energetically favored. The two plots are based on a $W=3$ and $L=4$ ribbon. The wavy bonds cross the periodic boundary, each of which connects two occupied sites generating an interacting energy $V$.

pair of adjacent bosons generating an interaction $V$. A domain wall along a direction other than the ribbon is shown in Fig. 17(b). It crosses three nonvertical bonds, and there appear three pairs of adjacent bosons with an interacting energy $3 \mathrm{~V}$. Generally when a domain wall crosses the nonvertical bonds for $n$ times, there appear $n$ pairs of adjacent bosons, and the interacting energy increases by $n V$. So for large interactions, a domain wall with turns is not energetically favored.

Although the domain walls with turns cannot be generated by the $\mathrm{NN}$ interactions, they can be designed using staggered potentials. We create a Z-shaped domain wall using staggered potentials with the strength $|\Delta|=4 t$ on a $W=12$ and $L=24$

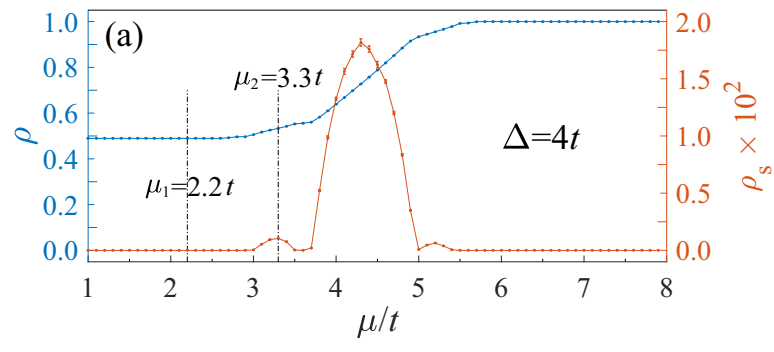

(b)

$$
\delta \rho_{i}=\rho_{i}\left(\mu_{2}\right)-\rho_{i}\left(\mu_{1}\right)
$$

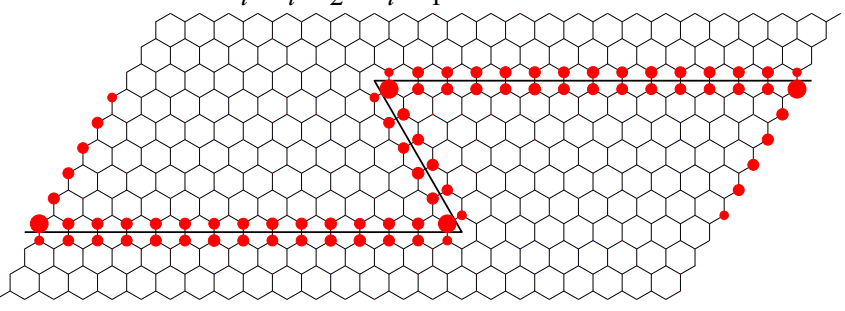

FIG. 18. (a) The average density and superfluid density as a function of $\mu$ on a ribbon with a Z-shaped domain wall generated using a staggered potential. (b) The distribution of the bosons added to the $\rho=\frac{1}{2}$ insulator. Ribbon width $W=12$ and length $L=24$. The strength of the staggered potential is $\Delta=4 t$.

ribbon, which is periodic along the longer axis. Figure 18(a) plots the average density and the superfluid density as a function of $\mu$. The $\rho=\frac{1}{2}$ plateau with vanishing superfluid corresponds to the domain-wall insulator. As the chemical potential further increases, bosons are added to the $\rho=\frac{1}{2}$ insulator. The superfluid density has finite values, and the system becomes a superfluid. We calculate the distribution of the added bosons, $\delta \rho_{i}=\rho_{i}\left(\mu_{2}\right)-\rho_{i}\left(\mu_{2}\right)$, with $\mu_{2}, \mu_{1}$ marked in Fig. 18(a). As shown in Fig. 18(b), the added bosons mainly distribute near the Z-shaped domain wall, implying the superfluid above the $\rho=\frac{1}{2}$ transporting down such a domain wall. With this method, complex-shaped domain walls can be designed, realizing circuits of superfluids.
[1] J. M. Tranquada, AIP Conf. Proc. 1550, 114 (2013).

[2] J. Zaanen and O. Gunnarsson, Phys. Rev. B 40, 7391 (1989).

[3] S. R. White and D. J. Scalapino, Phys. Rev. Lett. 91, 136403 (2003).

[4] C. S. Hellberg and E. Manousakis, Phys. Rev. Lett. 83, 132 (1999).

[5] E. Fradkin, S. A. Kivelson, and J. M. Tranquada, Rev. Mod. Phys. 87, 457 (2015).

[6] B.-X. Zheng, C.-M. Chung, P. Corboz, G. Ehlers, M.-P. Qin, R. M. Noack, H. Shi, S. R. White, S. Zhang, and G. K.-L. Chan, Science 358, 1155 (2017).

[7] E. W. Huang, C. B. Mendl, S. Liu, S. Johnston, H.-C. Jiang, B. Moritz, and T. P. Devereaux, Science 358, 1161 (2017).

[8] H.-C. Jiang and T. P. Devereaux, Science 365, 1424 (2019).

[9] I. Bloch, J. Dalibard, and W. Zwerger, Rev. Mod. Phys. 80, 885 (2008).

[10] M. A. Cazalilla, R. Citro, T. Giamarchi, E. Orignac, and M. Rigol, Rev. Mod. Phys. 83, 1405 (2011).
[11] B. Bauer, L. D. Carr, H. G. Evertz, A. Feiguin, J. Freire, S Fuchs, L. Gamper, J. Gukelberger, E. Gull, S. Guertler, A. Hehn, R. Igarashi, S. V. Isakov, D. Koop, P. N. Ma, P. Mates, H. Matsuo, O. Parcollet, G. Pawłowski, J. D. Picon, L. Pollet, E. Santos, V. W. Scarola, U. Schollwock, C. Silva, B. Surer, S. Todo, S. Trebst, M. Troyer, M. L. Wall, P. Werner, and S. Wessel, J. Stat. Mech.: Theory Exp. (2011) P05001.

[12] O. F. Syljuåsen and A. W. Sandvik, Phys. Rev. E 66, 046701 (2002).

[13] F. Alet, S. Wessel, and M. Troyer, Phys. Rev. E 71, 036706 (2005).

[14] L. Pollet, S. M. A. Rombouts, K. Van Houcke, and K. Heyde, Phys. Rev. E 70, 056705 (2004).

[15] S. R. White, Phys. Rev. Lett. 69, 2863 (1992).

[16] S. Wessel, Phys. Rev. B 75, 174301 (2007).

[17] T. Nakafuji and I. Ichinose, Phys. Rev. A 96, 013628 (2017).

[18] J. Y. Gan, Y. C. Wen, J. Ye, T. Li, S.-J. Yang, and Y. Yu, Phys. Rev. B 75, 214509 (2007). 
[19] A. H. Castro Neto, F. Guinea, N. M. R. Peres, K. S. Novoselov, and A. K. Geim, Rev. Mod. Phys. 81, 109 (2009).

[20] M. Fujita, K. Wakabayashi, K. Nakada, and K. Kusakabe, J. Phys. Soc. Jpn. 65, 1920 (1996).

[21] K. Nakada, M. Fujita, G. Dresselhaus, and M. S. Dresselhaus, Phys. Rev. B 54, 17954 (1996).

[22] Y.-W. Son, M. L. Cohen, and S. G. Louie, Phys. Rev. Lett. 97, 216803 (2006).

[23] W. Yao, S. A. Yang, and Q. Niu, Phys. Rev. Lett. 102, 096801 (2009).

[24] "Charge density wave" refers to coherent periodic modulation of hard-core bosons.

[25] E. L. Pollock and D. M. Ceperley, Phys. Rev. B 36, 8343 (1987).

[26] V. G. Rousseau, Phys. Rev. B 90, 134503 (2014).

[27] C.-Y. Hou, C. Chamon, and C. Mudry, Phys. Rev. Lett. 98, 186809 (2007).

[28] D. L. Bergman, Phys. Rev. B 87, 035422 (2013).

[29] Here the bosons are used to express the spin $-1 / 2$ operators, and they are distinct from hard-core bosons in Eq. (1).

[30] S. A. Owerre, J. Phys.: Condens. Matter 28, 436003 (2016).

[31] H. Guo, Y. Niu, S. Chen, and S. Feng, Phys. Rev. B 93, 121401(R) (2016).

[32] T. Fukui, Y. Hatsugai, and H. Suzuki, J. Phys. Soc. Jpn. 74, 1674 (2005).

[33] G. W. Semenoff, V. Semenoff, and F. Zhou, Phys. Rev. Lett. 101, 087204 (2008).

[34] S.-g. Cheng, H. Liu, H. Jiang, Q.-F. Sun, and X. C. Xie, Phys. Rev. Lett. 121, 156801 (2018).
[35] G. G. Batrouni, F. Hébert, and R. T. Scalettar, Phys. Rev. Lett. 97, 087209 (2006).

[36] M. Miyazaki, C. Hotta, S. Miyahara, K. Matsuda, and N. Furukawa, J. Phys. Soc. Jpn. 78, 014707 (2009).

[37] D. Jaksch, C. Bruder, J. I. Cirac, C. W. Gardiner, and P. Zoller, Phys. Rev. Lett. 81, 3108 (1998).

[38] M. Polini, F. Guinea, M. Lewenstein, H. C. Manoharan, and V. Pellegrini, Nat. Nanotechnol. 8, 625 (2013).

[39] S.-L. Zhu, B. Wang, and L.-M. Duan, Phys. Rev. Lett. 98, 260402 (2007).

[40] P. Soltan-Panahi, J. Struck, P. Hauke, A. Bick, W. Plenkers, G. Meineke, C. Becker, P. Windpassinger, M. Lewenstein, and K. Sengstock, Nat. Phys. 7, 434 (2011).

[41] D. Suszalski and J. Zakrzewski, Phys. Rev. A 94, 033602 (2016).

[42] T. Ozawa and H. M. Price, Nat. Rev. Phys. 1, 349 (2019).

[43] C. Gross and I. Bloch, Science 357, 995 (2017).

[44] I. Bloch, J. Dalibard, and S. Nascimbene, Nat. Phys. 8, 267 (2012).

[45] W. Bakr, J. Gillen, A. Peng, S. Folling, and M. Greiner, Nature (London) 462, 74 (2009).

[46] J. F. Sherson, C. Weitenberg, M. Endres, M. Cheneau, I. Bloch, and S. Kuhr, Nature (London) 467, 68 (2010).

[47] L. Duca, T. Li, M. Reitter, I. Bloch, M. Schleier-Smith, and U. Schneider, Science 347, 288 (2015).

[48] N. Fläschner, B. S. Rem, M. Tarnowski, D. Vogel, D.-S. Lühmann, K. Sengstock, and C. Weitenberg, Science 352, 1091 (2016).

[49] F. F. Assaad and I. F. Herbut, Phys. Rev. X 3, 031010 (2013). 